\section{Analysis of patients with chronic cerebro-spinal venous insufficiency and multiple sclerosis: identification of parameters of clinical severity}

\author{
Sandro Mandolesi, ${ }^{1}$ Aldo d'Alessandro, ${ }^{2}$ \\ Marco Matteo Ciccone, ${ }^{3}$ Annapaola Zito, ${ }^{3}$ \\ Ettore Manconi, ${ }^{4}$ Tarcisio Niglio, ${ }^{5}$ \\ Augusto Orsini, ${ }^{6}$ Dimitri Mandolesi, ${ }^{7}$ \\ Alessandro d'Alessandro, ${ }^{8}$ Francesco Fedele ${ }^{1}$ \\ 'Department of Cardio-vascular and \\ Respiratory Sciences, La Sapienza \\ University, Roma; ' ${ }^{2}$ epartment of \\ Neuroscience, Imaging and Clinical \\ Sciences, Chieti-Pescara University; \\ ${ }^{3}$ Cardiovascular Diseases Section, \\ Department of Emergency and Organ \\ Transplantation (DETO), University of \\ Bari; ${ }^{4}$ Department of Cardiovascular and \\ Neurological Sciences, University of \\ Cagliari; ${ }^{5}$ Italian National Health \\ Institute, Roma, Italy; ${ }^{6}$ Department of \\ Vascular Surgery, Gioia Hospital, Sora \\ (FR); ${ }^{7}$ Medicina del Lavoro, La Sapienza \\ University, Roma, Italy; ${ }^{8}$ Faculty of \\ Medicine, Foggia, Italy
}

\section{Abstract}

The aims of this study were: i) analysis of clinical severity evolution in multiple sclerosis patients; ii) identification of temporal indicators for clinical worsening. We investigated by echo-color-Doppler (ECD) 789 patients (490 female plus 299 male), aged 45.4 years, with chronic cerebro-spinal venous insufficiency (CCSVI) and multiple sclerosis (MS). All patients tested positive for CCSVI by ECD assessment were divided into three groups, namely: type 1 CCSVI (371) presenting an endo-vascular obstacle to the venous drainage; type 2 CCSVI (40) presenting an extra-vascular obstacle to the venous drainage, for external compression of the vessel; type 3 CCSVI (315) presenting both venous endo-vascular and extra-vascular obstructed drains. We analyzed the morphological and hemodynamic data recorded on computerized map (MEM-net). Al data were collected by respecting the Italian Privacy Laws and they are available on the National Epidemiological Observatory on CCSVI website (www.osservatorioccsvi.org). We focused in the three main parameters in all studied patients. First parameter was expanded disability status scale (EDSS) score; second parameter was illness duration; third parameter was CCSVI type. The MS duration values stratified by EDSS grouped values in CCSVItype- 1 and CCSVI-type-3 patients shows that the differences were statistical significant by Kruskal-Wallis test: $\mathrm{H}=44.2829$; degree of freedom $=1$ for CCSVI-type-1 $(\mathrm{P}<0.001)$; and $\mathrm{H}=37.3036$; degree of freedom $=1$ for CCSVItype-3 $(\mathrm{P}<0.001)$. The present study confirmed and completed scientific literature about relation between CCSVI and MS. On the same time, we found a strong correlation between MS illness duration and severity of EDSS score. In fact there is a clinical severity worsening after 11 years of illness in MS patients with CCSVI type-1 or type-3 $(\mathrm{P}<0.001)$. These data may suggest the influence of chronic vascular disease on MS. Further searches need in order to learn more about this new aspect in MS etiology.

\section{Introduction}

Multiple sclerosis (MS) is an inflammatory demyelinating disease with autoimmune pathogenesis that affects the central and peripheral nervous system, causing a variety of clinical signs and symptoms; ${ }^{1}$ these are mainly due to scar formation (better known as plaques or lesions) that form in the white matter of the spinal cord and brain., ${ }^{2,3}$

It is estimated that this disease has a prevalence that ranges between 2 and 150 cases per 100,000 individuals. ${ }^{4}$ It is believed that multiple sclerosis is an immune-mediated disease caused by a complex interaction between genetic subset of the individual and not yet identified environmental factors. ${ }^{5}$

In 2008, Paolo Zamboni noted that multiple sclerosis is related to altered vasculature where the cervical and thoracic veins are not able to efficiently remove blood from the central nervous system presumably because of stenosis and malformations of jugular veins and azygos. ${ }^{6-8}$ Therefore, he identified it as chronic cerebro-spinal venous insufficiency (CCSVI) and diagnosed it on the basis of hemodynamic alterations in relation to postural changes of the extra-and intracranial veins studied by Doppler sonography. ${ }^{9}$ According to Zamboni, CCSVI itself would facilitate ferrous cerebral accumulation, to which the body would respond with an immune reaction. ${ }^{10}$

Zamboni discovered this condition in most of people affected with multiple sclerosis, and after performing a surgical procedure to correct the problem stated that $73 \%$ of patients had achieved improvements.

The aim of this study was to analyze the evolution of the profiles of clinical severity in a sample of patients with multiple sclerosis and CCSVI with the intent to identify any temporal indicators of clinical worsening.
Correspondence: Sandro Mandolesi, via San Montebello 17, 00185, Roma, Italy. Tel.: +39.335.6512303 - Fax: +39.06.4873984 E-mail: s.mandolesi@email.it

Key words: multiple sclerosis, chronic cerebrospinal venous insufficiency, echo-color-Doppler.

Received for publication: 12 July 2014.

Revision received: 4 March 2015.

Accepted for publication: 5 March 2015.

This work is licensed under a Creative Commons Attribution 3.0 License (by-nc 3.0).

(C)Copyright S. Mandolesi et al., 2015

Licensee PAGEPress, Italy

Veins and Lymphatics 2015; 4:4570

doi:10.4081/vl.2015.4570

\section{Materials and Methods}

We assessed by echo-color-Doppler method (ECD) 789 patients with multiple sclerosis, their diagnosis was made in compliance with McDonald's revised criteria. ${ }^{11,12}$ All patients were assessed in the Department of Cardiovascular, Respiratory, Geriatric and Morphologic Sciences of Polyclinic Umberto I of Rome. Among the patients, 490 were female, 299 were male, with an overall mean age of 45.4 years. A total of 728 patients tested positive for CCSVI, while 61 were negative. In addition the morphological and hemodynamic ECD data were analyzed using a computerized map. This is a hemodynamic and morphological map named MEM-net, adopted after the Consensus Conference of the national scientific societies of vascular area to share the symbols and terminology to use for writing MEM-net mapping for a shared and standardized database. This allows us to stratify the data in the different degrees of venous disease, providing answers on the likely impact of CCSVI in MS and the various treatment options. All data were collected by respecting the Italian Privacy Laws and available on the National Epidemiological Observatory on CCSVI website (www.mem-net.it).

All patients tested positive for CCSVI by ECD assessment were divided into three groups, namely: i) patients affected by type 1 CCSVI (371) presenting an endo-vascular obstacle to the venous drainage, with congenital or acquired abnormalities that restrict and block the drainage of the investigated veins; ii) patients affected by type 2 CCSVI (40) presenting an extra-vascular obstacle to the venous drainage, for external compression of the vessel; iii) patients affected by type 3 CCSVI (315) presenting both venous endo-vascular and extra-vascular obstructed drains.

In order to simplify this classification, we 
can say that there is a hydraulic CCSVI (type 1), a mechanical CCSVI (type 2) and a mixed CCSVI of the previous two types (type 3 ). The method used to diagnose the presence of an obstacle due to external compression of the vessel (CCSVI type 2) and not by narrowing the inside of the same (CCSVI type 1) was a simple intuition: through the Valsalva maneuver and appropriate rotation of the head by the examined subject, it was possible to note a significant change in the crossing section area and the reappearance of the flow within the studied vessel We have presented in 2011 at the National Congress of the Italian Society of Cardiology the division into 3 types of CCSVI. ${ }^{13,14}$

We focused three main parameters to stratify all studied patients for parametric and nonparametric statistical tests. First parameter was CCSVI-type; second parameter was ill duration; third parameter was expanded disability status scale (EDSS) total score.

In our sample the CCSVI-type- 2 patients were few in number for statistical tests; so we analyzed only CCSVI-type-1 and CCSVI-type-3 patients. After the analysis of variance (ANOVA), we divided all ill duration into two groups (before and after the median value): less than 12 years (1-11 years of illness) and more than 11 years (12 or more).

EDSS total scores were analyzed as natural values (see Kurtzke, ${ }^{15}$ for details) or as grouped values. We stratified EDSS total scores into three categories: 123 or mild impairment (EDSS total score: 1 or 2 or 3); 456 or medium impairment (EDSS total score: 4 or 5 or 6); 789 or severe impairment (EDSS total score: 7 or 8 or 9 ).

In our tests, we considered only mild (123) and severe (789) grouped EDSS values. Medium status of illness (456 grouped EDSS values) was not considered to increase strength in differences between initial and final MS period.

\section{Diagnosis of chronic cerebro-spinal venous insufficiency by echo-color- Doppler}

In order to make a diagnosis of CCSVI, according to Zamboni's protocol, it is necessary that the patient has at least 2 of the following 5 parameters: i) reflux in internal jugular veins and/or vertebral veins in the supine and sitting position; reflux disease is defined as a reverse flow that lasts more than $0.88 \mathrm{sec}$ onds: ${ }^{16}$ ii) reflux in intracranial veins (internal cerebral vein, basal vein of Rosenthal, and great cerebral vein of Galen); iii) presence of stenosis in the internal jugular vein using Bmode high-resolution echography; in this regard it is possible to identify hemodynamically (intraluminal defects such as fixed valves and/or malformed valves, spider webs, septa) or hemodynamically presence of stenosis in the internal jugular vein B-mode high-resolution in this regard it is possible to identify stenosis hemodynamically (intraluminal defects such as fixed valves and/or malformed, spider webs, septa) or not- hemodynamically significant stenosis; ${ }^{17}$ iv) no detectable flow using Doppler in the internal jugular veins and/or vertebral veins; v) reverse postural control of the main cerebral venous outflow pathways by evaluating the $\Delta$ cross sectional area $(\Delta \mathrm{CSA})$ in the internal jugular vein, that is the difference between the circumference of the internal jugular vein in the supine and sitting position, measured at its midpoint, ${ }^{18-22}$ The finding of a negative CSA indicates a loss of postural control of the predominant way of venous drainage in the supine position.

During the ECD assessment before the patient is positioned in the supine position and then in a sitting position; the examination is conducted with both quiet breathing (without moving shoulders, to activate the respiratory pump and check if there is an induction of cerebro-spinal drainage) and deep breathing (to induce the maximum flow in the vessels which must be examined and for assessing the correct drainage and valvular functioning). In all our ECD assessments we performed the following ECD dynamic tests: neck movements, on right, on left rotation and anterior/posterior intrusion of the neck; Valsalva's maneuver, performed by moderately forceful attempted exhalation against a closed airway, usually done by closing one's mouth and pinching one's nose shut.

The protocol requires the use of two probes: a 6.6-10 MHz linear probe, which is necessary for scanning the veins of the neck and a transcranial probe from 2.0 to $3.3 \mathrm{MHz}$ for the study of the intracranial circulation. It can optionally be used a micro-convex probe from 5.0 to 8.0 $\mathrm{MHz}$ that allows to make a more accurate assessment of the lower part of the neck vessels, i.e. jugular-subclavian ostium and intrarachidian veins. ${ }^{7}$

\section{Statistical analysis}

All data were analyzed by SPSS software to perform a stratified data description for numeric parametric variables. Statistical significance between and within groups was calculated on continuous variables by ANOVA to test the equality of means. The Chi-square $\left(\chi^{2}\right)$ Yates corrected test was used for non-continuous variables by Statcalc and Analysis programs from Epi-Info (2008, NIH \& CDC Atlanta, GA, USA; Italian version 3.5.1).

A p value less than 0.05 was considered significant, and $95 \%$ confidence intervals were also calculated. The collected data of this study were assessed by the Kruskal-Wallis statistical test that is the non-parametric equivalent test of the analysis of variance in which the data are replaced by their rank; it was used because the studied population did not follow a normal Gaussian distribution. This non-parametric method has the purpose of comparing the equality of medians in the different groups.

\section{Results}

In Table 1 we show the cross tabulation between EDSS grouped values vs MS duration (less or more than 11 years) in CCSVI-type-1 and CCSVI-type-3 patients. The differences were statistical significant $(\mathrm{P}<0.001)$.

In Table 2 we show the ANOVA of EDSS values stratified by MS duration in CCSVI-type-1 and CCSVI-type-3 patients. The differences were statistical significant by Kruskal-Wallis test: $H=8.0345$; degree of freedom $=1$ for CCSVI-type-1 $(\mathrm{P}<0.01)$; and $\mathrm{H}=8.4757$; degree of freedom=1 for CCSVI-type-3 $(\mathrm{P}<0.01)$.

In Table 3 we show the ANOVA of MS duration values stratified by EDSS grouped values in CCSVI-type-1 and CCSVI-type-3 patients. The differences were statistical significant by Kruskal-Wallis test: $\mathrm{H}=44.2829$; degree of freedom=1 for CCSVI-type-1 $(\mathrm{P}<0.001)$; and $\mathrm{H}=37.3036$; degree of freedom $=1$ for CCSVItype-3 $(\mathrm{P}<0.001)$.

In Table 4 we show the ANOVA of EDSS values stratified by CCSVI-type-1 or CCSVI-type-3 patients in MS duration values (less or more than 11 years). The differences relived were not statistical significant.

If we consider instead the EDSS profiles of three clinical types we find that the relapsingremitting (RR) patients are more present in the period before 11 years, the secondary progressive (SP) are more frequent after 11 years and the primary progressive (PP) uniformly distributed in the pre-and post-11 years.

\section{Discussion}

The present study confirmed the data on the prevalence of CCSVI in MS patients, already mentioned in previous papers. In a large study, which involved 6 centers, Bastianello et al. analyzed the clinical data of 710 MS patients who underwent venous echo-color-Doppler. Significant associations were found between CCSVI and clinical data. ${ }^{23}$ Similar results were quite recently described by an active multicenter epidemiological registry. ${ }^{24}$ Therefore, our results replicate those coming from developed international scientific literature about this topic, as well as from several confirming metaanalysis. $^{25}$ Recently it was also shown that diagnostic procedure by ECD is reproducible between different operators from the technical perspective. ${ }^{26}$ In recent years several studies have dealt with the association between CCSVI 
Table 1. Cutoff in chronic cerebro-spinal venous insufficiency-type-1 and -type-3 patients.

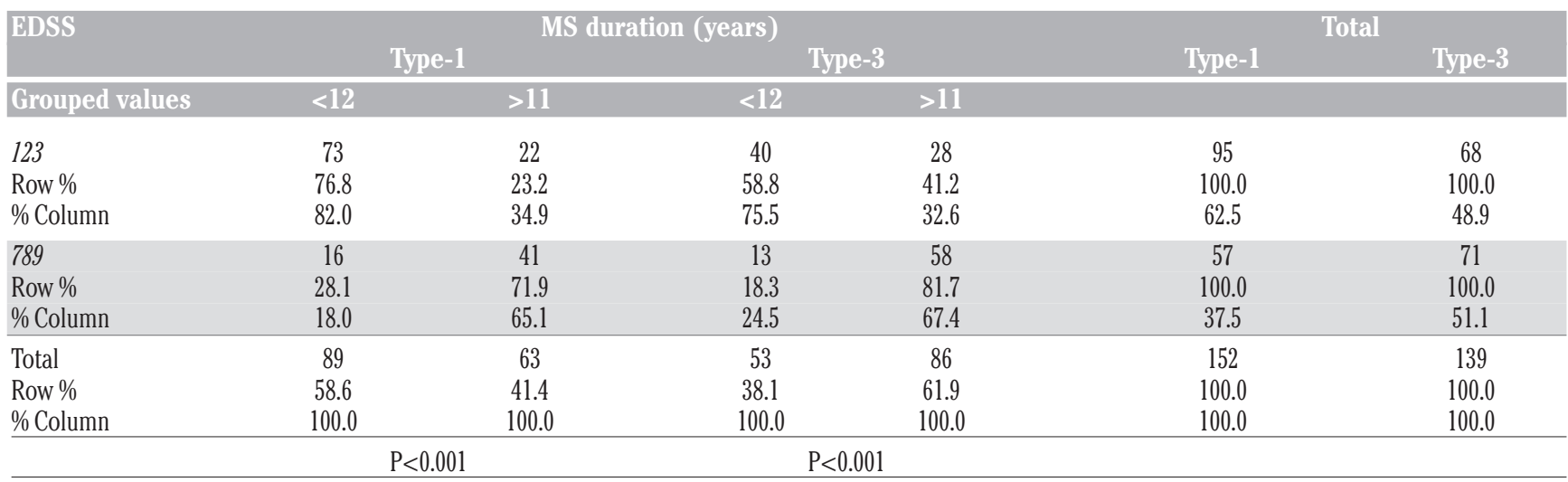

EDSS, expanded disability status scale; MS, multiple sclerosis.

Table 2. Expanded disability status scale values in cerebro-spinal venous insufficiency-type-1 and -type-3 patients.

\begin{tabular}{|c|c|c|c|c|c|c|c|c|c|c|c|c|}
\hline \multirow{2}{*}{$\begin{array}{l}\text { MS duration } \\
\text { (years) }\end{array}$} & \multicolumn{2}{|c|}{ Obs } & \multicolumn{2}{|c|}{$\Sigma$} & \multicolumn{2}{|c|}{ Mean } & \multicolumn{2}{|c|}{ Variance } & \multicolumn{4}{|c|}{ Std.Dev. } \\
\hline & Туре-1* & Type-3 ${ }^{\circ}$ & Туре-1* & Type-$^{\circ}{ }^{\circ}$ & Type-1* & Type- $^{\circ}{ }^{\circ}$ & Туре-1* & Type- $3^{\circ}$ & Type- $1^{*}$ & Type- $3^{\circ}$ & & \\
\hline$<12$ & 16 & 15 & 630.000 & 735.000 & 39.375 & 49.000 & 62.625 & 64.000 & 25.025 & 25.298 & & \\
\hline$>11$ & 26 & 25 & 1600.000 & 1705.000 & 61.538 & 68.200 & 43.954 & 24.767 & 20.965 & 15.737 & & \\
\hline & \multicolumn{2}{|c|}{ Minimum } & \multicolumn{2}{|c|}{$25 \%$} & \multicolumn{2}{|c|}{ Median } & \multicolumn{2}{|c|}{$75 \%$} & \multicolumn{2}{|c|}{ Maximum } & Moc & \\
\hline & Туре-1* & 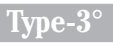 & Туре-1* & Type- $3^{\circ}$ & Туре-1* & Type- $3^{\circ}$ & Туре-1* & Type-3 ${ }^{\circ}$ & Type-1* & Туре--3 & Туре-1* & Type-3 $3^{\circ}$ \\
\hline$<12$ & 15.000 & 15.000 & 15.000 & 25.000 & 25.000 & 65.000 & 65.000 & 65.000 & 75.000 & 85.000 & 15.000 & 65.000 \\
\hline$>11$ & 15.000 & 15.000 & 65.000 & 65.000 & 65.000 & 75.000 & 75.000 & 75.000 & 85.000 & 85.000 & 65.000 & 75.000 \\
\hline
\end{tabular}

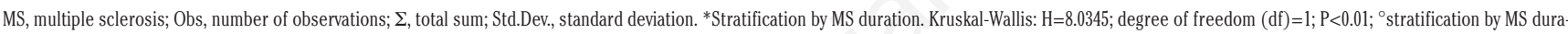
tion. Kruskal-Wallis: $\mathrm{H}=8.4757 ; \mathrm{df}=1 ; \mathrm{P}<0.01$.

Table 3. Multiple sclerosis duration values in cerebro-spinal venous insufficiency-type-1 and -type-3 patients.

\begin{tabular}{|c|c|c|c|c|c|c|c|c|c|c|c|}
\hline \multirow{2}{*}{$\begin{array}{l}\text { EDSS grouped } \\
\text { values }\end{array}$} & \multicolumn{2}{|c|}{ Obs } & \multicolumn{2}{|c|}{$\Sigma$} & \multicolumn{2}{|c|}{ Mean } & \multicolumn{2}{|c|}{ Variance } & \multicolumn{2}{|c|}{ Std.Dev. } & \\
\hline & Туре-1* & Type- $3^{\circ}$ & Type-1* & Type- $3^{\circ}$ & Type-1* & Type- $^{\circ}$ & Type-1* & Type- $3^{\circ}$ & Туре- $1^{*}$ & Type- $3^{\circ}$ & \\
\hline 123 & 95 & 68 & 742.0000 & 733.0000 & 7.8105 & 10.7794 & 44.0701 & 58.2640 & 6.6385 & 7.6331 & \\
\hline \multirow[t]{3}{*}{789} & 57 & 71 & 990.0000 & 1480.0000 & 17.3684 & 20.8451 & 84.1654 & 87.8471 & 9.1742 & 9.3727 & \\
\hline & \multicolumn{2}{|c|}{ Minimum } & \multicolumn{2}{|c|}{$25 \%$} & \multicolumn{2}{|c|}{ Median } & \multicolumn{2}{|c|}{$75 \%$} & \multicolumn{2}{|c|}{ Maximum } & ode \\
\hline & Type-1* & Type-3 & Type-1* & Type-3을 & Type-1* & Type- $^{\circ}$ & Type-1* & Type- $3^{\circ}$ & Type-1*T & pe-3 ${ }^{\circ}$ Type- $1^{*}$ & Type- $3^{\circ}$ \\
\hline 123 & 0.0000 & 0.0000 & 2.0000 & 4.5000 & 6.0000 & 9.0000 & 11.0000 & 16.0000 & 27.0000 & 36.00002 .0000 & 2.0000 \\
\hline 789 & 3.0000 & 5.0000 & 11.0000 & 14.0000 & 16.0000 & 21.0000 & 22.0000 & 28.0000 & 46.0000 & 50.000011 .0000 & 31.0000 \\
\hline
\end{tabular}

EDSS, expanded disability status scale; Obs, number of observations; $\Sigma$, total sum; Std.Dev., standard deviation. *Stratification by EDSS grouped values. Kruskal-Wallis: $\mathrm{H}=44.2829$; degree of freedom (df)=1; $\mathrm{P}<0.001 ;{ }^{\circ}$ stratification by EDSS grouped values. Kruskal-Wallis: $\mathrm{H}=37.3036 ; \mathrm{df}=1 ; \mathrm{P}<0.001$.

Table 4. Expanded disability status scale values in patients with multiple sclerosis duration $<12$ and $>11$ years.

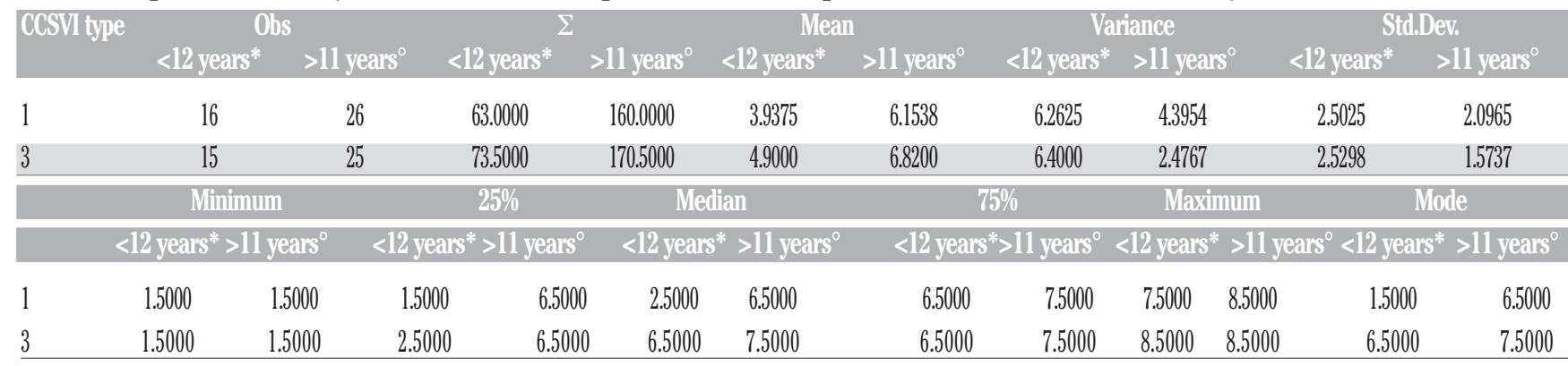

CCSVI, chronic cerebro-spinal venous insufficiency; Obs, number of observations; $\Sigma$, total sum; Std.Dev., standard deviation. *Stratification by CCSVI-type. Kruskal-Wallis: H=1.2725; degree of freedom (df) $=1$; not significant; ${ }^{\circ}$ stratification by CCSVI-type. Kruskal-Wallis: $\mathrm{H}=2.5481$; df=1; not significant. 
and MS. Among these, very interesting is the work conducted by Zivadinov and co-workers, who described the effective association between the neurodegenerative disease (MS) and this functional-hemodynamic finding which affects the vessels draining the cerebral blood. Furthermore they have shown that the higher disability the higher the prevalence of CCSVI is in MS patients. In particular, it emerged that the prevalence of CCSVI was higher in patients with progressive MS than in those with non-progressive MS. Therefore the authors concluded with the assumption that a high probability of disease progression which was associated with a high level of clinical disability was induced by morphological and hemodynamic alterations of cerebral veins. ${ }^{27}$ In our previous study we have also found that patients aged more than 30 years showed a statistically significant higher presence of disabilities (mean EDSS score equal to 5) compared to younger patients (mean EDSS score equal to 3). ${ }^{28}$ The same considerations may be made in present study, in which we highlighted that MS clinical severity worsening in CCSVI patients after 11 years of disease duration $(\mathrm{P}<0.001)$.

On 2009 Zamboni suggested that the RR and SP clinical courses are associated with significantly different CCSVI patterns compared to those with PP form. ${ }^{7}$

In the same paper, Zamboni suggest that location of venous obstructions could play a key role in determining the clinical course of the disease. Yamout et al., however, have subsequently stated that the extra-cranial venous stenosis are unlikely the cause of MS, since in their study they were not present in most patients in the early stages of the disease and rarely involved more than one extra-cranial vein. They supposed, therefore, that CCSVI was a late secondary phenomenon. ${ }^{29}$ Several authors have contrasted the initial hypothesis of Zamboni, for example Baracchini et al., in their work, have shown that CCSVI is not a late secondary phenomenon of MS and is not associated with the worsening of disability. ${ }^{30}$ Baracchini himself, in 2011, did not support a cause-effect relationship between CCSVI and MS onset. ${ }^{31}$ Centonze et al. in their work also found no statistically significant differences in the frequency of CCSVI in MS patients and controls. ${ }^{32}$ In addition, they have found no difference between patients CCSVI-positive and CCSVI-negative in terms of relevant clinical changes, such as the disease duration, the time that elapses between the onset and the first exacerbation, remitting or gradual course of disease and the risk of secondary progressive course. Another datum emerged from this study: there were no statistically significant differences between CCSVI-positive and CCSVI-negative MS patients in mean EDSS, mean index of progression and mean score of severity of MS. No significant correlation was found between the severity of the disability and the number of positive criteria for CCSVI. Their results therefore indicated that CCSVI has no role in either the risk or severity of MS.

In present work, we do not found statistical significant differences between the different types of CCSVI and the EDSS score in patients with the same MS duration. Patients with progressive MS had higher probability of having also CCSVI than those with RR MS.

These findings suggested that the presence of CCSVI may favor a late development of MS in patients with a lower susceptibility to autoimmune diseases and may increase its severity. Already, other studies have found scores of clinical severity generally higher in CCSVI-positive patients. ${ }^{33}$ Recently, some papers stated that CCSVI has no effect on neurological function and on the progression of disability in patients with MS. ${ }^{34}$ Other casecontrol clinical works ${ }^{35}$ showed that CCSVI was more frequent among MS patients with an ill duration more than 144 months. The same clinical aspects were found in SP MS and PP MS versus RR MS patients. A stronger association was found considering the forms SP and PP of MS. The authors therefore showed a higher frequency of CCSVI in MS patients, more evident in patients with advanced disease, suggesting that CCSVI may be related to disability in MS. Still about the study of the association between CCSVI and clinical disability in MS, Leone et al. ${ }^{36}$ evaluated the association of CCSVI with MS in a cross-sectional blinded study and sought some association of CCSVI with the prevalence of MS. The prevalence of CCSVI was related to age in MS patients but not in controls. CCSVI-positive and CCSVI-negative patients were similar in clinical type, age at onset of illness, disability and fatigue. The disease duration was greater in CCSVI-positive patients than in negative ones. The authors concluded that CCSVI was not associated with MS, nor its severity. In addition, they could not affirm that CCSVI is a consequence of MS or aging. From this examination of the literature we understand how there are different and conflicting opinions about the correlation between CCSVI and MS, especially with regard to its relationship with the age from first clinical evidence, the degree of ill progression and patient capabilities deterioration.

\section{Conclusions}

Present study confirmed and complete previous papers about relation between CCSVI and MS. On the same time, we found a strong correlation between MS illness duration and severity of EDSS score. In fact there is a clini- cal severity cut-off after 11 years of illness in MS patients with CCSVI-type-1 or CCSVI-type3. On the contrary, we did not found statistical significant differences comparing MS patients with CCSVI-type-1 versus MS patients with CCSVI-type-3. If we consider instead the EDSS profiles of three clinical types we find that the RR patients are more present in the period before 11 years, the SP are more frequent after 11 years and the PP uniformly distributed in the pre-and post-11 years. These data may suggest chronic vascular disease influence on MS. Further searches need in order to learn more about this new aspect in MS etiology.

\section{References}

1. Compston A, Coles A. Multiple sclerosis. Lancet 2008;372:1502-17.

2. Clanet M. Jean-Martin Charcot. 1825 to 1893. Int MS J 2008;15:59-61.

3. Charcot J. Histologie de la sclerose en plaques. Gazette des Hopitaux, Paris 1868;41:554-5.

4. Rosati G. The prevalence of multiple sclerosis in the world: an update. Neurol Sci 2001;22:117-39.

5. Compston A, Coles A. Multiple sclerosis. Lancet 2002;359:1221-31.

6. Zamboni P, Galeotti R. The chronic cerebrospinal venous insufficiency syndrome. Phlebology 2010;25:269-79.

7. Zamboni P, Galeotti R, Menegatti E, et al. Chronic cerebro-spinal venous insufficiency in patients with multiple sclerosis. J Neurol Neurosurg Psychiatry 2009;80: 392-9.

8. Radak D, Kolar J, Tanaskovic S, et al. Morphological and haemodynamic abnormalities in the jugular veins of patients with multiple sclerosis. Phlebology 2012; 27:168-72.

9. Zamboni P, Morovic S, Menegatti E, et al. Screening for chronic cerebrospinal venous insufficiency (CCSVI) using ultrasound - recommendations for a protocol. Int J Angiol 2011;30:571-97.

10. Singh AV, Zamboni P. Anomalous venous blood flow and iron deposition in multiple sclerosis. J Cereb Blood Flow Metab 2009;29:1867-78.

11. Polman CH, Reingold SC, Edan G, et al. Diagnostic criteria for multiple sclerosis: 2005 revisions to the "McDonald Criteria". Ann Neurol 2005;58:840-6.

12. McDonald WI, Compston A, Edan G, et al. Recommended diagnostic criteria for multiple sclerosis: guidelines from the International Panel on the diagnosis of multiple sclerosis. Ann Neurol 2001;50: 121-7.

13. Mandolesi S, Ciciarello F, Marceca A, et al. 
Data analysis of the chronic cerebro-spinal venous insufficiency in patients with multiple sclerosis: new disease classification. $72^{\circ}$ Congresso Nazionale della Società Italiana di Cardiologia, 10-12 Dicembre 2011, Roma, Italy.

14. Mandolesi S, Manconi E, Niglio T, et al. Incidence of anatomical compressions of the internal jugular veins with full block of their flow in patients with chronic cerebro-spinal venous insufficiency and multiple sclerosis. In: Allegra C, Antignani PL, eds. Proc. 21st EUROCHAP-IUA - European Chapter Congress of the International Union of Angiology. Sept 28-Oct 1, 2013, Rome, Italy. Turin: Ed. Minerva Medica; 2013. pp 12-17.

15. Kurtzke JF. Rating neurologic impairment in multiple sclerosis: an expanded disability status scale (EDSS) Neurology 1983;33:1444-52.

16. Valdueza JM, Schmierer K, Mehraein S, Einhaupl KM. Assessment of normal flow velocity in basal cerebral veins. A transcranial Doppler ultrasound study. Stroke 1996;27:1221-5.

17. Lepori D, Capasso P, Fournier D, et al. High-resolution ultrasound evaluation of internal jugular venous valves. Eur Radiol 1999;9:1222-6.

18. Schaller B. Physiology of cerebral venous blood flow: from experimental data in animals to normal function in humans. Brain Res Rev 2004;46:243-60.

19. Valdueza, JM, von Munster T, Hoffman 0, et al. Postural dependency of the cerebral venous outflow. Lancet 2000;355:200-1.

20. Gisolf, J, van Lieshout JJ, van Heusden K, et al. Human cerebral venous outflow pathway depends on posture and central venous pressure. J Physiol 2004;560:317-27.

21. Schreiber SJ, Lurtzing F, Gotze R, et al. Extrajugular pathways of human cerebral venous blood drainage assessed by duplex ultrasound. J Appl Physiol 2003;94:1802-5.

22. Menegatti E, Zamboni P. Doppler haemodynamics of cerebral venous return. Curr Neurovasc Res 2008;5:260-5.

23. Bastianello S, Romani A, Viselner G, et al. Chroniccerebrospinalvenousinsufficiency in multiple sclerosis: clinicalcorrelates from a multicentrestudy. BMC Neurol 2011;11:132.

24. Mandolesi S, d'Alessandro A, Ciccone MM, et al. Italian Chronic Cerebrospinal Venous Insufficiency National Epidemiological Observatory methodology and preliminary data. Veins and Lymphatics 2014;3:4707.

25. Zamboni P, Menegatti E, Occhionorelli S, Salvi F. The controversy on chronic cerebrospinal venous insufficiency. Veins and Lymphatics 2013;2:e14.

26. Ciccone MM, Galeandro AI, Scicchitano P, et al. Multigate quality Doppler profiles and morphological/hemodynamic alterations in multiple sclerosis patients. Curr Neurovasc Res 2012;9:120-7.

27. Zivadinov R, Marr K, Cutter G, et al. Prevalence, sensitivity, and specificity of chronic cerebrospinal venous insufficiency in MS. Neurology 2011;77:138-44.

28. Ciciarello F, Mandolesi S, Galeandro AI, et al. Age-related vascular differences among patients suffering from multiple sclerosis. Curr Neurovasc Res 2014;11:23-30.
29. Yamout B, Herlopian A, Issa Z, et al. Extracranial venous stenosis is an unlikely cause of multiple sclerosis. Mult Scler 2010;16:1341-8.

30. Baracchini C, Perini P, Causin F, et al. Progressive multiple sclerosis is not associated with chronic cerebrospinal venous insufficiency. Neurology 2011;77:844-50.

31. Baracchini C, Perini P, Calabrese M, et al. No evidence of chronic cerebrospinal venous insufficiency at multiple sclerosis onset. Ann Neurol 2011;69:90-9.

32. Centonze D, Floris R, Stefanini M, et al. Proposed chronic cerebrospinal venous insufficiency criteria do not predict multiple sclerosis risk or severity. Ann Neurol 2011;70:51-8.

33. Weinstock-Guttman B, Cutter G, Marr K, et al. Clinical correlates of chronic cerebrospinal venous insufficiency in multiple sclerosis. Multiple Sclerosis 2010;16:S224.

34. Garaci FG, Marziali S, Meschini A, et al. Brain hemodynamic changes associated with chronic cerebrospinal venous insufficiency are not specific to multiple sclerosis and do not increase its severity. Radiology 2012;265:233-9.

35. Patti F, Nicoletti A, Leone C, et al. Multiple sclerosis and CCSVI: a population-based case control study. PloS One 2012;7: e41227.

36. Leone MA, Raymkulova 0, Nald PI, et al. Chronic cerebrospinal venous insufficiency is not associated with multiple sclerosis and its severity: a blind-verified study. PloS One 2013;8:e56031. 